\title{
Photonic Skin for Pressure and Strain Sensing
}

\author{
Xianfeng Chen ${ }^{* a}$, C. Zhang ${ }^{\mathrm{a}}$, B. Van Hoe ${ }^{\mathrm{b}}$,

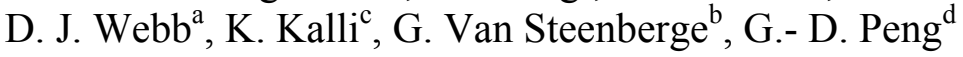 \\ ${ }^{a}$ School of Engineering and Applied Science, Aston University, Birmingham B4 7ET, UK; \\ ${ }^{\mathrm{b}}$ Centre for Microsystems Technology, Gent University, B-9052 Zwijnaarde, Belgium; \\ ${ }^{\mathrm{c}}$ Nanophotonics Research Laboratory, Cyprus University of Technology, Cyprus; \\ ${ }^{\mathrm{d}}$ School of Electrical Engineering, University of New South Wales, Australia
}

\begin{abstract}
In this paper, we report on the strain and pressure testing of highly flexible skins embedded with Bragg grating sensors recorded in either silica or polymer optical fibre. The photonic skins, with a size of $10 \mathrm{~cm} \mathrm{x} 10 \mathrm{~cm}$ and thickness of $1 \mathrm{~mm}$, were fabricated by embedding the polymer fibre or silica fibre containing Bragg gratings in Sylgard 184 from Dow Corning. Pressure sensing was studied using a cylindrical metal post placed on an array of points across the skin. The polymer fibre grating exhibits approximately 10 times the pressure sensitivity of the silica fibre and responds to the post even when it is placed a few centimetres away from the sensing fibre. Although the intrinsic strain sensitivities of gratings in the two fibre types are very similar, when embedded in the skin the polymer grating displayed a strain sensitivity approximately 45 times greater than the silica device, which also suffered from considerable hysteresis. The polymer grating displayed a near linear response over wavelength shifts of $9 \mathrm{~nm}$ for $1 \%$ strain. The difference in behaviour we attribute to the much greater Young's modulus of the silica fibre (70 GPa) compared to the polymer fibre (3 GPa).
\end{abstract}

Keywords: Photonic skin, optical fibre, Bragg grating, polymer optical fibre, sensor, pressure, strain, loading

\section{INTRODUCTION}

The demonstration that fibre Bragg gratings (FBGs) can be written in polymer optical fibre (POF) has extended the possibilities for grating based devices for optical communications and optical sensing applications ${ }^{1}$. The development of this technology is motivated by the rather different mechanical and chemical properties of polymeric materials compared to silica ${ }^{2}$, which may be advantageous for certain applications. To-date applications research has been frustrated by the availability of FBGs only at long wavelengths, predominantly $1550 \mathrm{~nm}$, where the fibre attenuation is very large and fibre lengths must consequently be short $(<10 \mathrm{~cm})$. This problem has been partially overcome by the development of permanent glued connections between silica fibre and polymer fibre allowing the polymer FBG to be used away from the optical bench for the first time ${ }^{3}$.

Within project PHOSFOS (PHOtonic Skins For Optical Sensing), we have been working to develop the technology needed to realize flexible sensing skins incorporating optics and processing electronics ${ }^{4}$. As part of this activity, we have been comparing the strain and pressure sensing performance of FBG sensors in silica and polymer fibre when embedded in an elastic skin; the results are described in this paper.

\section{INSCRIPTION OF FIBRE BRAGG GRATINGS}

The POF used in our experiments is a single mode step index polymethylmethacrylate (PMMA) based fibre with a core diameter of $9.5 \mu \mathrm{m}$ and a cladding diameter of $200 \mu \mathrm{m}$. The polymer FBG was inscribed by the scanning phase mask technique using a CW helium-cadmium laser (Kimmon model IK5652R-G) with an output wavelength of $325 \mathrm{~nm}$, a beam diameter of $1.8 \mathrm{~mm}$ and a power of $30 \mathrm{~mW}$. As shown in figure 1, with the polymer fibre mounted horizontally in a v-groove, the laser beam was focused vertically down onto the fibre axis using a cylindrical lens of focal length $10 \mathrm{~cm}$.

Optical Sensing and Detection, edited by Francis Berghmans, Anna Grazia Mignani, Chris A. van Hoof, Proc. of SPIE Vol. 7726, 772604 - (C) 2010 SPIE · CCC code: 0277-786X/10/\$18 · doi: 10.1117/12.854235 
A phase mask with period $1057 \mathrm{~nm}$ was placed on top of the fibre to generate a periodic intensity modulation in the core region. The laser beam was scanned $6 \mathrm{~mm}$ along the phase mask during the approximately 30 minute recording time. The inscription process was monitored by butt-coupling the angle-cleaved end of a single mode silica fibre coupler to the POF using a drop of index matching oil, the end of the POF having previously been cleaved using a razor blade on a hot plate at $80^{\circ} \mathrm{C}^{5}$. Using the coupler, the POF was illuminated with broadband light covering the range $1530 \mathrm{~nm}$ to 1610 $\mathrm{nm}$ (Thorlabs, Broadband ASE light source) and the reflection spectrum monitored using an optical spectrum analyser (OSA, HP86142A).

The silica FBG was made in hydrogen-loaded corning SMF-28 fibre using a frequency doubled argon ion laser at 244 $\mathrm{nm}$ wavelength with a $1071.9 \mathrm{~nm}$ period phase mask. The laser beam was scanned along the phase mask once to produce a grating $3 \mathrm{~mm}$ in length. After the UV exposure, the silica $\mathrm{FBG}$ was annealed at $85^{\circ} \mathrm{C}$ for $24 \mathrm{hrs}$ to remove residual hydrogen and stabilise its optical properties.

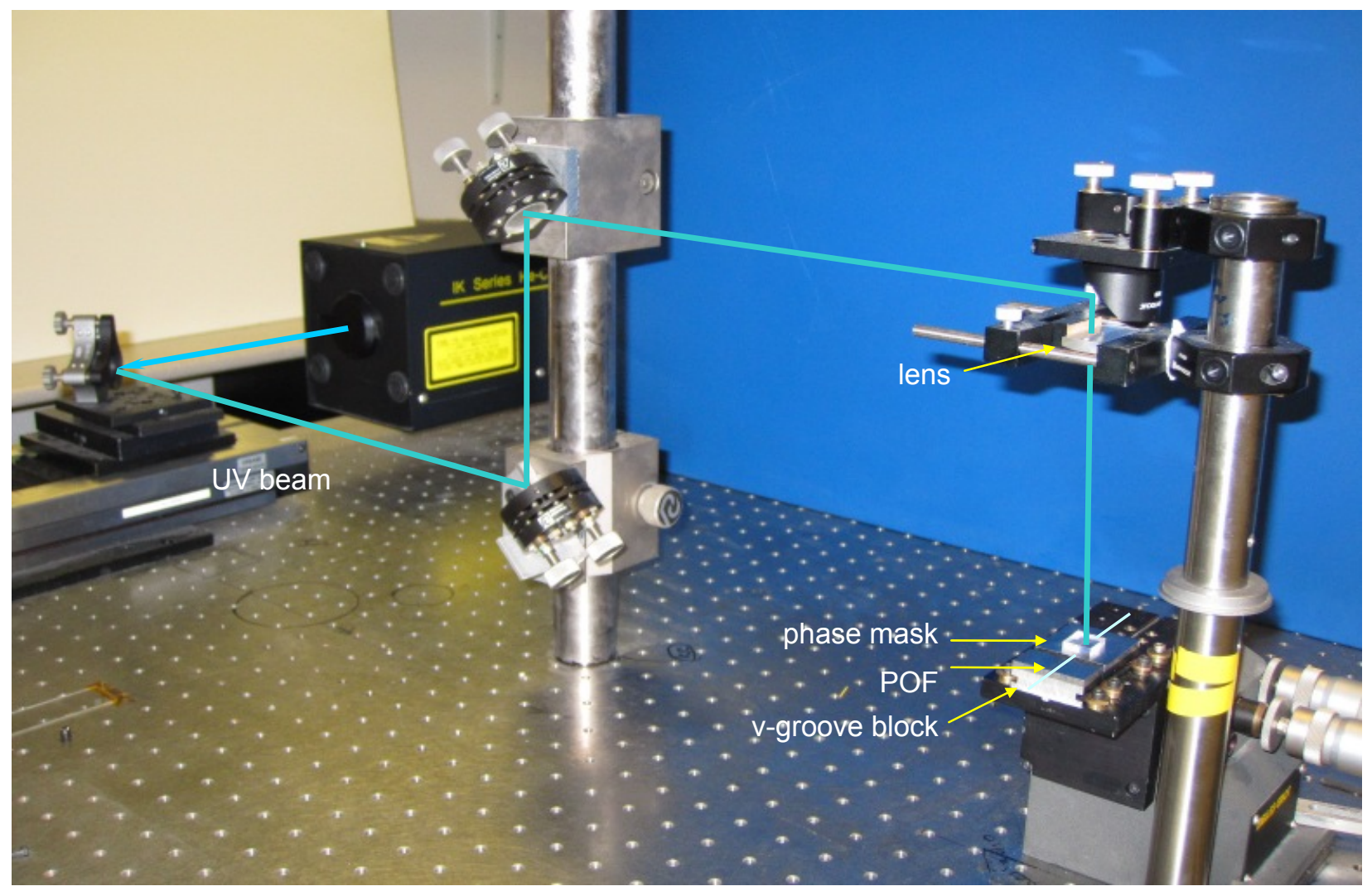

Figure 1. Experimental arrangement for FBG fabrication in polymer optical fibre.

Following its inscription, the polymer fibre containing the $6 \mathrm{~mm}$-long Bragg grating in the middle section was glued to a single mode silica fibre pigtail using UV curing optical adhesive (NOA 76, Norland Products, Inc.) cured with the OmniCure Series 1000 (EXFO Photonic Solution Inc.) . The grating reflection spectrum after completion of the gluing process is plotted in figure 2, and shows a Bragg peak at $1564.03 \mathrm{~nm}$ and a reflective power above the noise level of $20 \mathrm{~dB}$. The combination of the Bragg wavelength and phase mask period can be used to determine that the effective index of the core mode was 1.459 . 


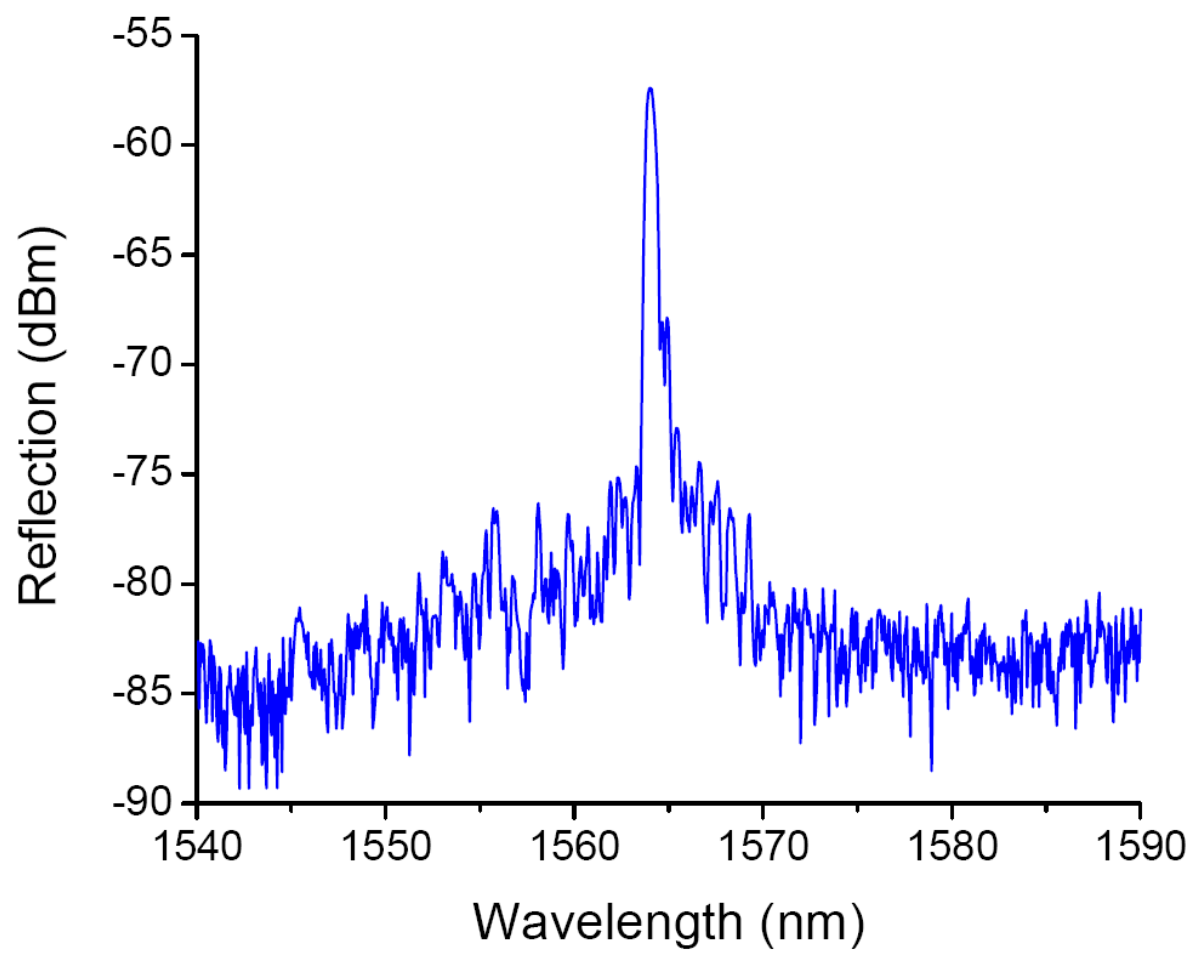

Figure 2. Reflection spectrum of polymer FBG glued with a single mode silica fibre pigtail. Resolution bandwidth $0.1 \mathrm{~nm}$.

\section{FABRICATION OF PHOTONIC SKINS}

The photonic skin was fabricated by embedding the entire $7.5 \mathrm{~cm}$-long POF with the POF-silica fibre gluing point in a stretchable host material (Sylgard 184 from Down Corning), with the FBG located in the centre of the square skin $(10 \mathrm{~cm} \times 10 \mathrm{~cm} \times 1 \mathrm{~mm}$, see Figure $3 \mathrm{a})$. The embedding process was performed using an iron frame in which the stretchable material was cured at a maximum temperature of $60^{\circ} \mathrm{C}$. For POF FBGs it is important that the temperature does not approach the glass transition temperature of about $104{ }^{\circ} \mathrm{C}$ or the grating will be erased ${ }^{6}$. In the case of the silica FBG, there was no glued connection within the skin, see Figure 4a. The reflection spectra of the FBGs were monitored before and after embedding in the stretchable material, with the results being shown in Figure $3 \mathrm{~b}$ and Figure $4 \mathrm{~b}$.

In the case of the silica FBG, the embedding process caused a blue shift of $0.84 \mathrm{~nm}$ (Figure $4 \mathrm{~b}$ ), which was probably due to the shrinkage of the host stretchable skin. This corresponds to an induced strain in the silica fibre of $700 \mu \varepsilon^{7}$. In the case of the polymer FBG, embedding induced a decrease in the Bragg wavelength of $10.75 \mathrm{~nm}$ (Figure $3 \mathrm{~b}$ ), which would correspond to an induced strain of $7.2 \mathrm{m \varepsilon}{ }^{1}$. In addition to any strain induced by the shrinkage of the skin, there is also likely to be a shift due to annealing of the $\mathrm{POF}^{6}$, which leads to shrinkage of the fibre, most likely due to a relaxation of the fibre molecular structure, partially aligned by the drawing process, back to a more disordered state. 


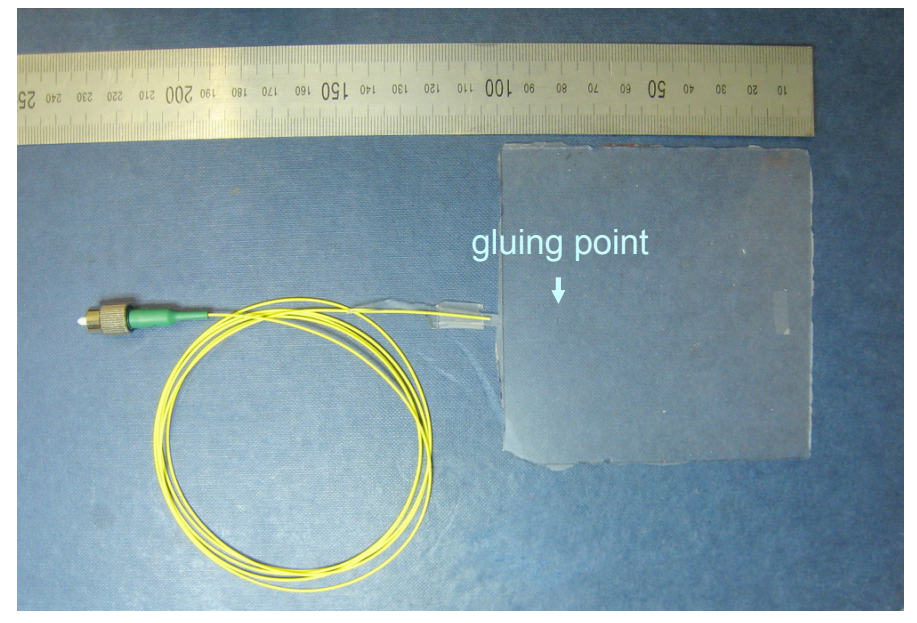

(a)

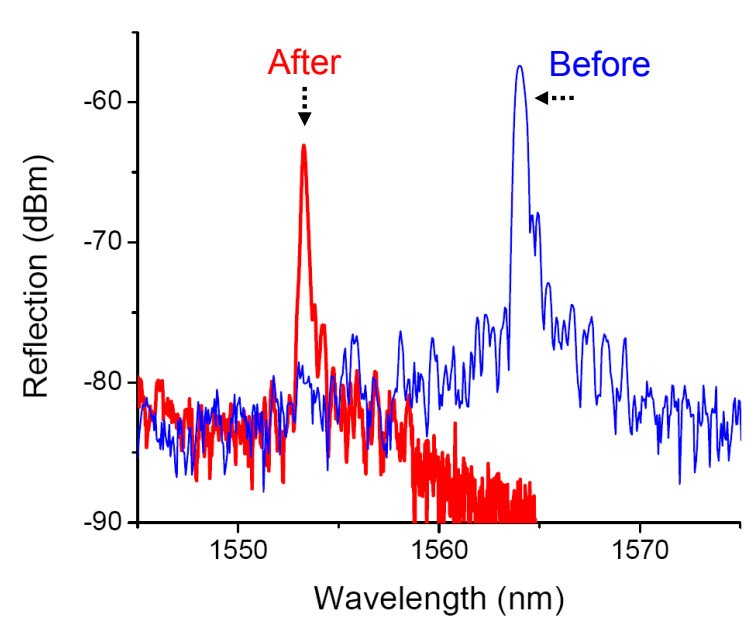

(b)

Figure 3. (a) Photonic skin embedded with polymer FBG;

(b) Polymer FBG spectra measured before and after the grating was embedded in photonic skin.

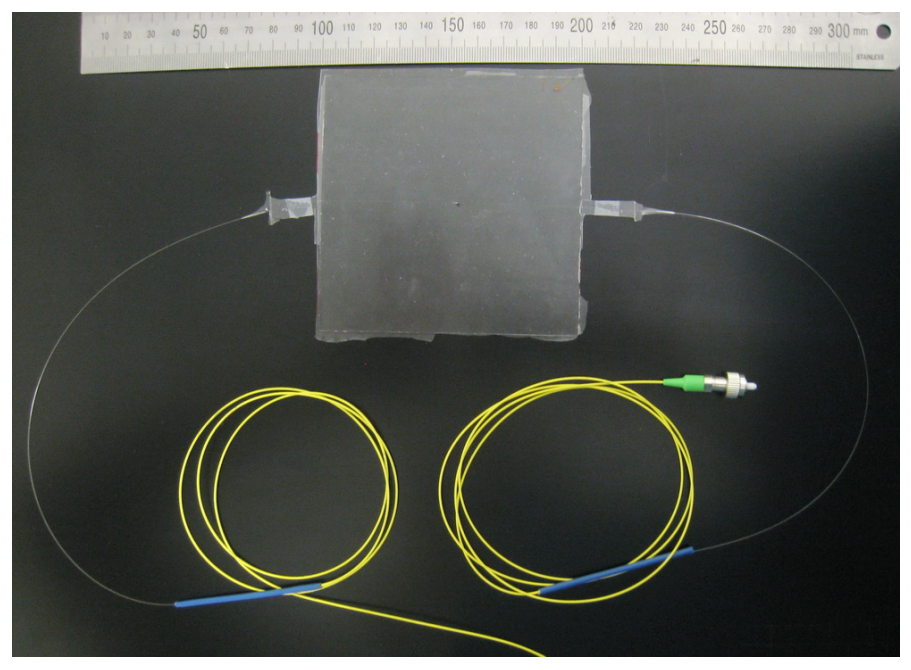

(a)

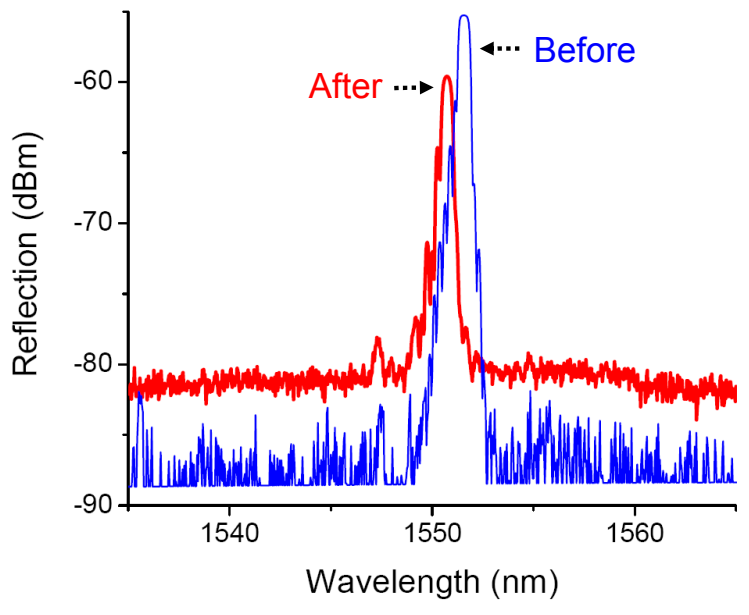

(b)

Figure 4. (a) Photonic skin embedded with silica FBG;

(b) Silica FBG spectra measured before and after the grating was embedded in photonic skin. 


\section{PRESSURE SENSING}

The sensitivity of the photonic skin to local squeezing (pressure) was measured with the setup as shown in figure 5 . The sample of the photonic skin was placed on the flat surface of the metal optical bench and the pressure was applied with a cylinder metal post (with a fixed mass of $74 \mathrm{~g}$ and a bottom diameter of $9 \mathrm{~mm}$ ). The line along the fibre underneath was set as the $\mathrm{x}$-axis with the point $[0,0]$ defines as the centre of the FBG. Several points along the $\mathrm{x}$-axis with $8 \mathrm{~mm}$ separation were selected to apply the pressure. The Bragg wavelength change in reflection was monitored with the optical spectral analyzer as the pressure was applied to the photonic skin. All the data have been plotted in figure 6 (for the POF FBG) and figure 7 (for the silica FBG case).

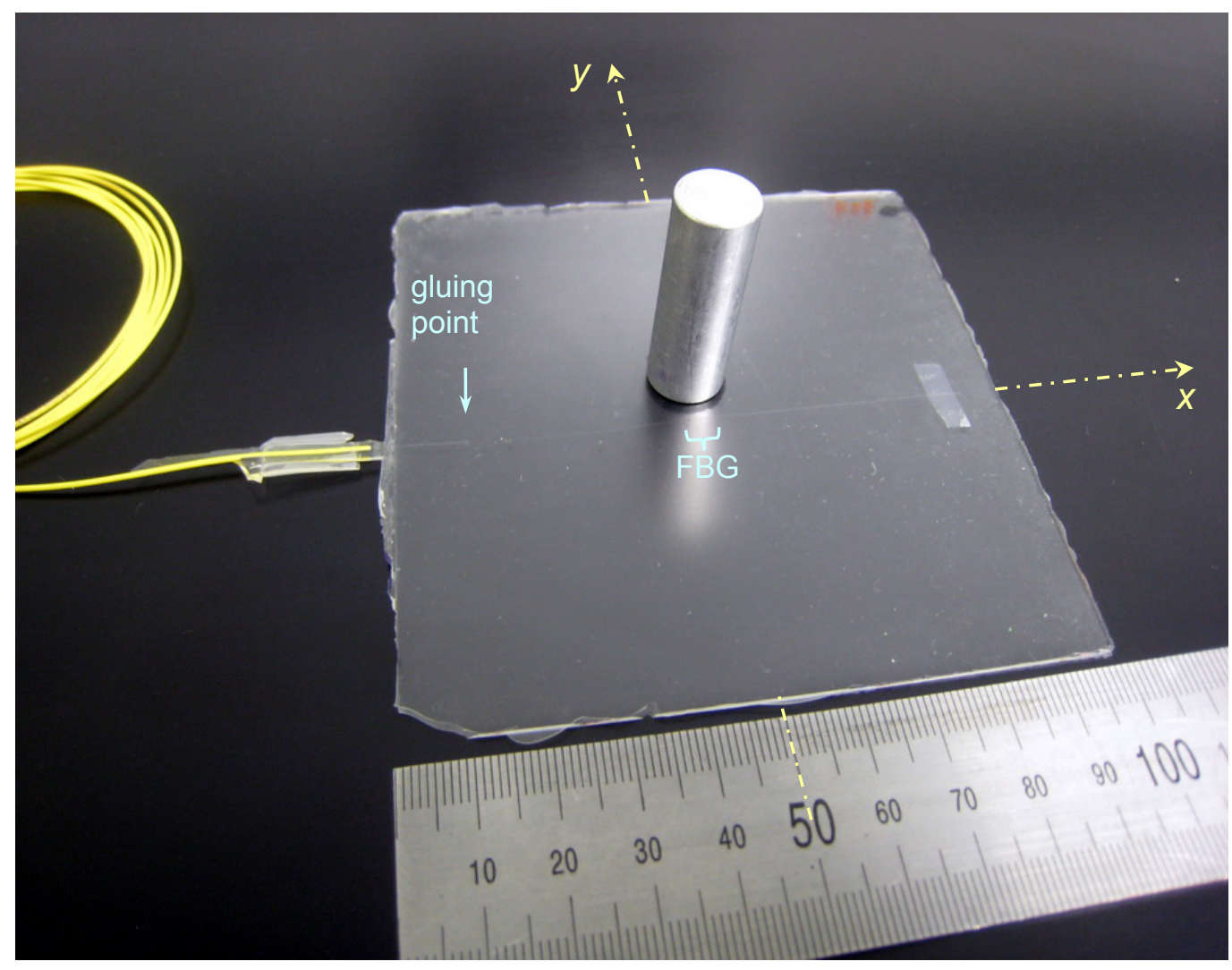

Figure 5. Schematic of pressure applied on the photonic skin.

The results show that not only the centre point $[0,0]$, with the FBG exactly underneath the post, but also all the neighbouring points along the $\mathrm{x}$-axis were sensitive to the applied pressure. It is clear that the Bragg peak moved to the long wavelength side for all the loaded points. Although the pressure was applied along the $\mathrm{x}$-axis with equal separation of $8 \mathrm{~mm}$, the Bragg wavelength shift shows a non-linear response with distance: the closer the post was placed to the centre point $[0,0]$, the larger the response got with the maximum pressure sensitivity occurring at point $[0,0]$.

The responses shown in figures 6 and 7 are qualitatively similar. The key difference is in the wavelength scale where it can be seen the POF FBG is a little over 10 times more sensitive to pressure than the silica device (wavelength shift of $519 \mathrm{pm}$ for POF as against 49pm for silica).. To compare figure 6 and figure 7, the wavelength shift of the polymer FBG displays a greater response to the pressure than the corresponding point of the silica FBG device. The difference in behaviour we attribute to the much greater Young's modulus of the silica fibre (70 GPa $)^{8}$ compared to the polymer fibre $(3 \mathrm{GPa})^{9}$. 


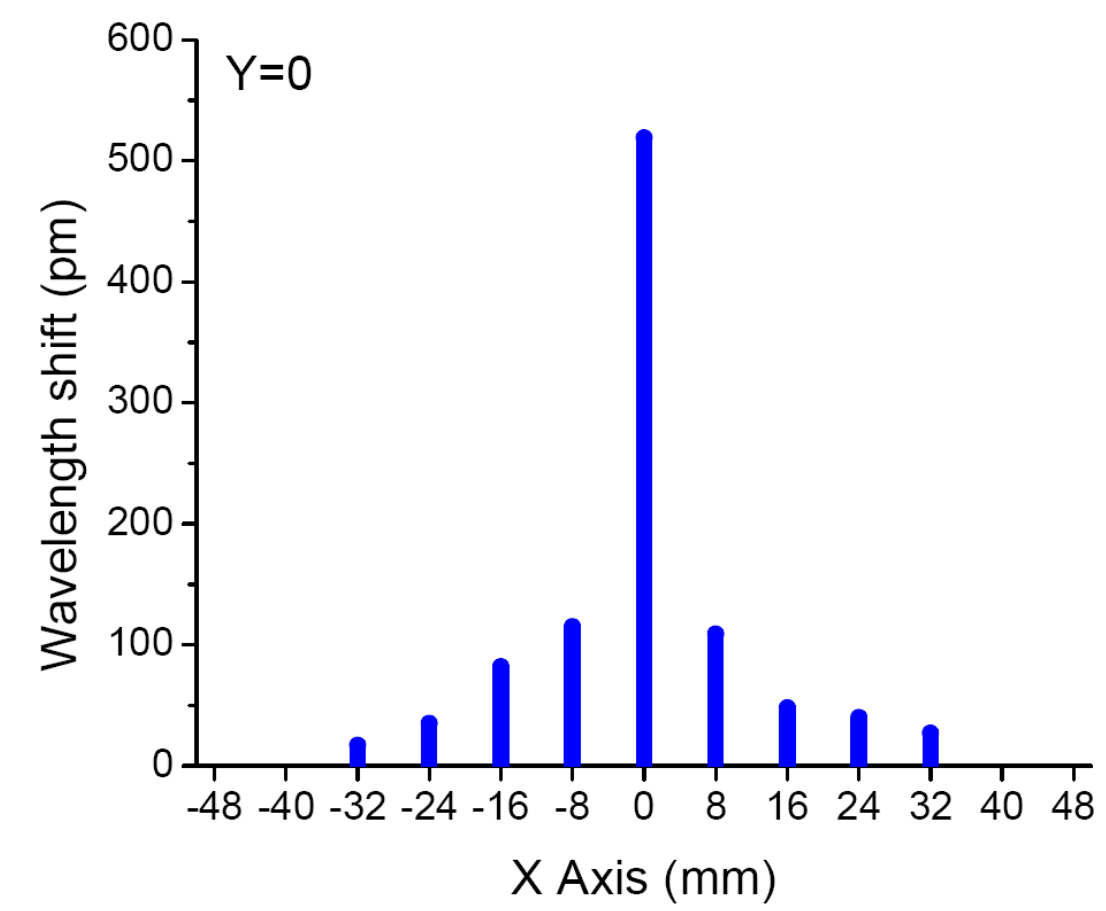

Figure 6. Wavelength responses caused by the pressure applied on polymer FBG photonic skin along the $\mathrm{x}$ axis $(\mathrm{y}=0)$ where the polymer fibre lies underneath.

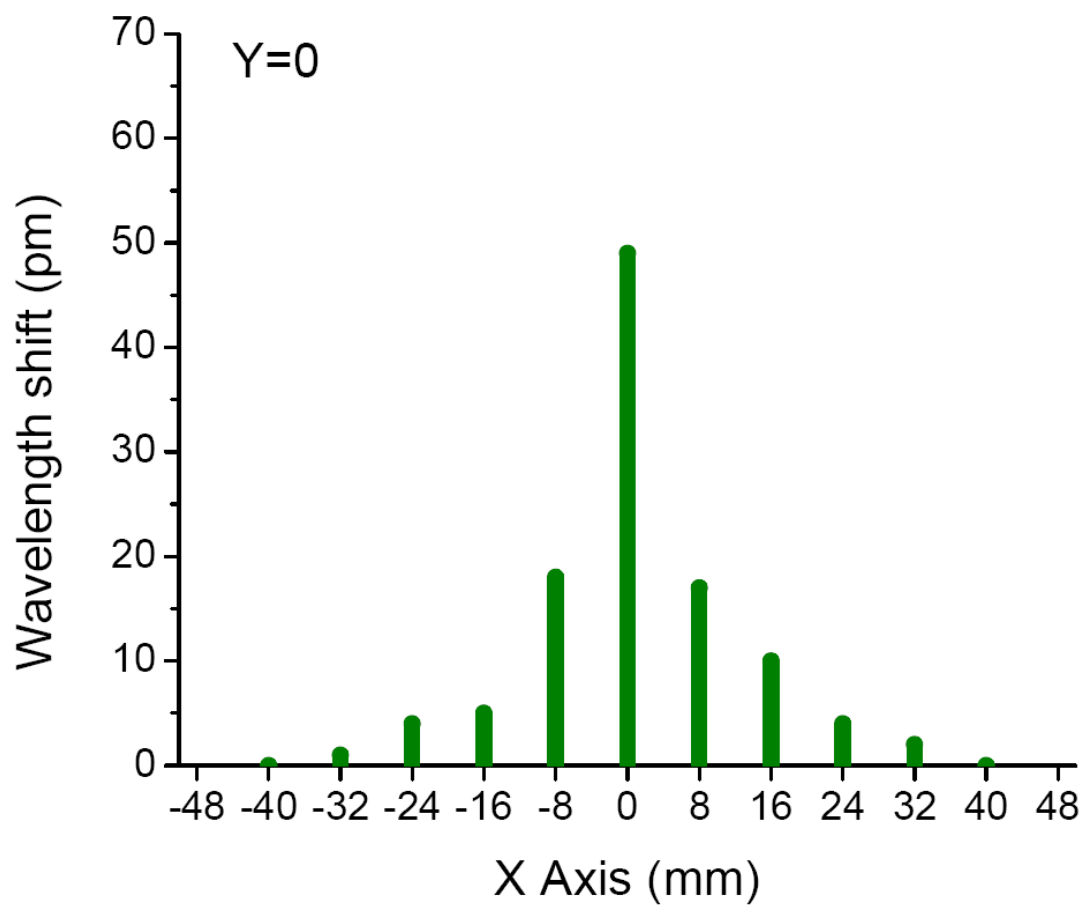

Figure 7. Wavelength responses caused by the pressure applied on silica FBG photonic skin along the $\mathrm{x}$ axis $(\mathrm{y}=0)$ where the silica fibre lies underneath. 


\section{STRAIN SENSING}

The sensitivities of the photonic skins to strain were studied with the experimental setup shown in figure 8 . To hold the skin, eight $2 \mathrm{~mm}$ diameter holes were melted into the skin to allow it to be clamped between aluminium plates attached to translation stages. A gap of $4 \mathrm{~mm}$ was left between the plates where the fibre was situated. The resulting testing arrangement had a gauge length of $50 \mathrm{~mm}$. Both skins were tested by repeatedly increasing the applied strain up to $1 \%$ after which the translation stage was returned to its starting position incrementally. The whole process took approximately 30 minutes.

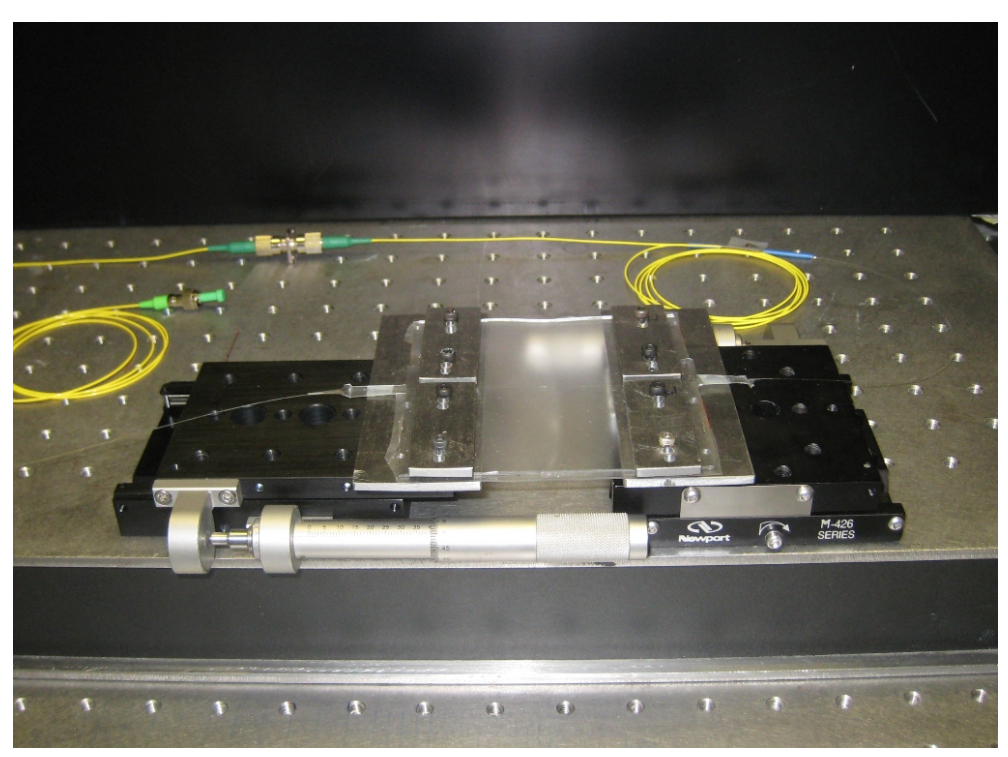

Figure 8. Experimental setup for photonic skin strain sensing

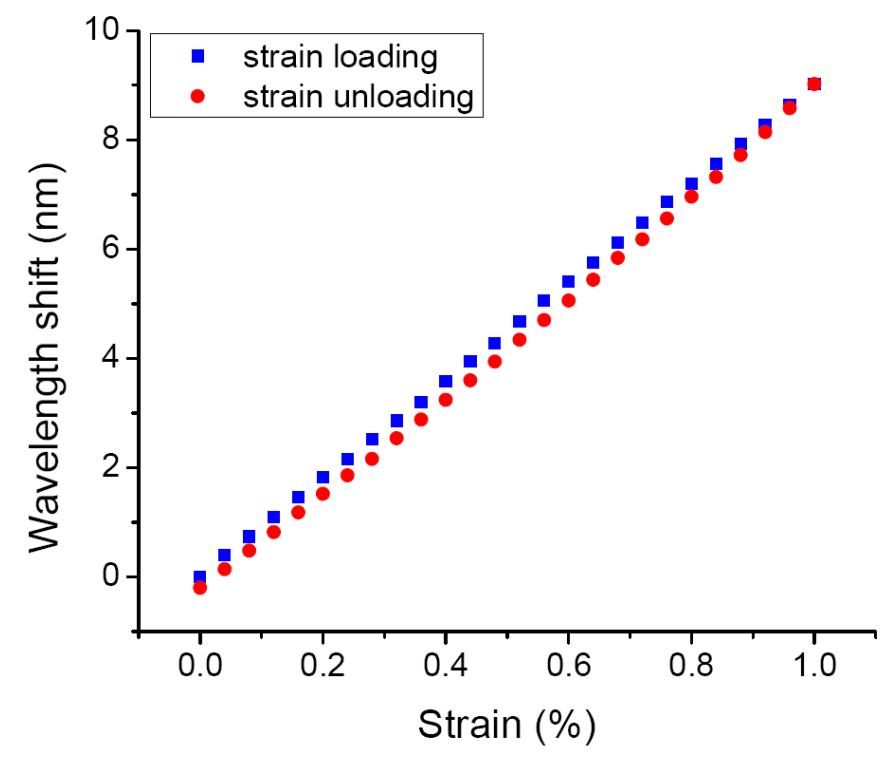

Figure 9. Strain behaviour of the polymer FBG embedded photonic skin with strain loading up to $1.0 \%$ and then unloading to the initial point. 
If we first consider the data for the skin containing the POF FBG shown in Figure 9, we can see the loading data exhibits good linearity, with a small amount of hysteresis apparent when the fibre is unloaded. It is unclear at the moment whether this hysteresis is due to the skin material or the fibre itself; certainly this kind of behaviour has been reported for POF ${ }^{10}$. The total wavelength shift at $1 \%$ strain was $9.02 \mathrm{~nm}$, which leads to a strain sensitivity of $0.9 \mathrm{pm} / \mu \varepsilon$. This value is a little smaller than that reported for bare POF, which is $1.5 \mathrm{pm} / \mu \varepsilon^{11}$. The difference here is likely to arise from the mismatch in elastic moduli of the fibre and skin; although PMMA is much less stiff than silica fibre, the Sylgard skin has an elastic modulus 3 orders of magnitude less ${ }^{12}$ and so the fibre is bound to locally reinforce the skin, reducing the strain in the fibre region.

The equivalent results for the silica fibre are shown in Figure 10. In several attempts it proved impossible to obtain data showing a smooth response and the data in the plot are typical. The results for loading and unloading are barely monotonic in places and there is considerable hysteresis visible. When the skin is unloaded back to the point where the net wavelength shift is zero, the translation stage still indicates a strain of $0.19 \%$. If the strain is removed the sensor exhibits a net wavelength shift of $-0.02 \mathrm{~nm}$. This may indicate that the fibre has slipped within the skin in places. At $1 \%$ applied strain, the total wavelength shift is $0.21 \mathrm{~nm}$, which corresponds to a strain sensitivity of just $0.021 \mathrm{pm} / \mu \varepsilon$, as compared to the bare-fibre sensitivity of $1.2 \mathrm{pm} / \mu \varepsilon^{7}$. Clearly the much stiffer silica fibre is less able to report the background strain in the skin.

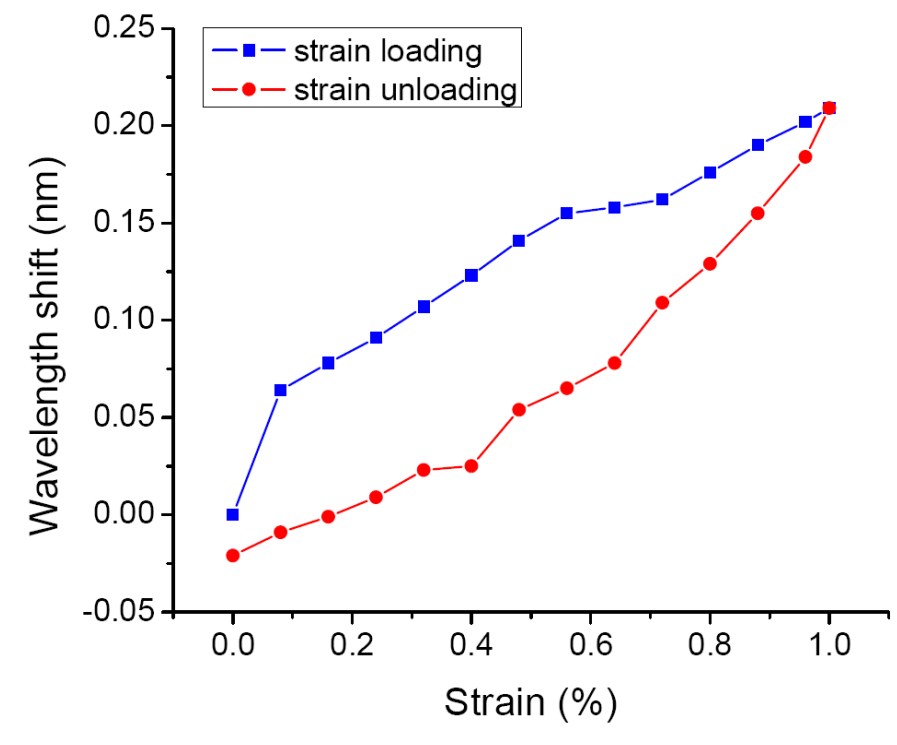

Figure 10. Strain behaviour of the silica FBG embedded photonic skin with strain loading up to $1 \%$ and then unloading to the initial point.

\section{CONCLUSION}

We have reported the results of experiments designed to study the pressure and strain sensitivity of highly flexible skins containing embedded silica and POF FBG sensors. The POF FBGs are more sensitive and report a strain value much closer to the background strain within the skin. They also exhibit much less hysteresis than the silica fibre in this configuration. Fibre Bragg gratings have been proposed as tactile sensors to create an artificial skin for robotics related applications ${ }^{13}$; the research reported here suggests that it is worth investigating the use of POF FBGs for such applications. 


\section{ACKNOWLEDGEMENTS}

The authors acknowledge the support of the European Commission through Project PHOSFOS (PHOtonic Skins For Optical Sensing).

\section{REFERENCES}

[1] Xiong, Z., Peng et al., "Highly tunable Bragg gratings in single-mode polymer optical fibers," IEEE Photonics Technology Letters, 11(3), 352-354 (1999).

[2] D. J. Webb, and K. Kalli, "Polymer fibre Bragg gratings" [Fiber Bragg Grating Sensors: Recent Advancements, Industrial Applications and Market Exploitation] Bentham eBooks, (2010).

[3] C. C. Ye, J. M. Dulieu-Barton, D. J. Webb et al., [Applications of polymer optical fibre grating sensors to condition monitoring of textiles], Edinburgh(2009).

[4] http://www.phosfos.eu/.

[5] S. H. Law, J. D. Harvey, R. J. Kruhlak et al., "Cleaving of microstructured polymer optical fibres," Optics Communications, 258(2), 193-202 (2006).

[6] K. E. Carroll, C. Zhang, D. J. Webb et al., "Thermal response of Bragg gratings in PMMA microstructured optical fibers," Optics Express, 15(14), 8844-8850 (2007).

[7] A. D. Kersey, M. A. Davis, H. J. Patrick et al., "Fiber grating sensors," Journal of Lightwave Technology, 15(8), 1442-1463 (1997).

[8] G. W. Kaye, and T. H. Laby, [Tables of physical and chemical constants] Longmann, 45 (1995).

[9] J. Brandrup, [Polymer Handbook] Wiley, V89 (1999).

[10] D. Yang, J. Yu, X. Tao et al., "Structural and mechanical properties of polymeric optical fiber," Materials Science and Engineering, A364, 256-259 (2004).

[11] G. D. Peng, and P. L. Chu, "Polymer optical fiber photosensitivities and highly tunable fiber gratings," Fiber and Integrated Optics, 19, 277-293 (2000).

[12] F. Schneider, T. Fellner, J. Wilde et al., "Mechanical properties of silicones for MEMS," Journal of Micromechanics and Microengineering, 18(6), (2008).

[13] J. S. Heo, J. H. Chung, and J. J. Lee, "Tactile sensor arrays using fiber Bragg grating sensors," Sensors and Actuators a-Physical, 126(2), 312-327 (2006). 